\title{
Temperature effect on light propagation in a tapered optical fiber with a twisted nematic liquid crystal cladding
}

\author{
Joanna Korec, ${ }^{*}$ Karol A. Stasiewicz, Leszek R. Jaroszewicz \\ Institue of Applied Physics, Military University of Technology, Kaliskiego 2, 00-908 Warsaw
}

Received November 28, 2008; accepted March 31, 2019; published March 31, 2019

\begin{abstract}
This paper presents the influence of temperature on the optical power spectrum propagated in a tapered optical fiber with a twisted nematic liquid crystal cladding (TOF-TNLCC) modulated by an electric field. Measurements were performed for a liquid crystal cell with a twisted orientation of ITO layers, filled with the E7 mixture. Induced reorientation of a liquid crystal (LC) n-director was measured for a visible and near-infrared wavelength range $[550 \div 1100 \mathrm{~nm}]$ in an electric field range of $0 \div 160 \mathrm{~V}$ and temperature range of $20 \div 60^{\circ} \mathrm{C}$. The relation between the temperature and the optical power spectrum of the investigated device was established.
\end{abstract}

Tapering is one of fiber optic technologies which allows producing various elements like couplers, insulators, polarizers, etc [1]. In most cases, this technique uses heat and slow elongation of optical fiber at its softening point to change the angle of taper transition as slightly as possible. A low losses structure is obtained for an adiabatic taper characterized by a long transition region compared to the taper waist region [2]. Since a light beam is propagated in the whole structure of a taper waist without division into core and cladding, hence it is possible to influence beam propagation inside the fiber by boundary conditions modification [3]. In a structure created in this way, the taper waist region becomes the core and the surrounding medium becomes its cladding.

The idea of surrounding optical fiber tapers with a liquid crystal was created by $\mathrm{Ch}$. Veilleux (et al.) and is

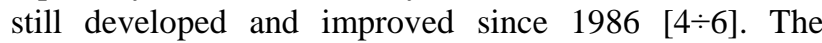
thickness of the first investigated cells was about $\sim 200 \mu \mathrm{m}$ and consists of electrodes without any alignment layer. The threshold voltage required to initiate the orientation of molecules in those structures reached up to 350V [4]. The cells prepared by the authors are 5 times thinner than the first ones and glass substrates are covered with ITO and alignment layers. Diameter reduction of the tapered waist allows to reduce the thickness of a liquid crystal cell, hence it is possible to decrease the threshold voltage to $40 \mathrm{~V}$ and obtain good projection of a steering signal. In this paper the authors used as cladding a nematic LC E7 mixture, which is characterized by a wide range of operating wavelength $\lambda=550 \div 1100 \mathrm{~nm}, \quad T_{c}=60^{\circ} \mathrm{C}$, $n_{e}=1.739, n_{o}=1.523, \varepsilon_{\perp}=6.0, \varepsilon_{\|}=19.3[7 \div 8]$. By steering

*E-mail: joanna.korec@wat.edu.pl the molecules orientation in an LC layer, a different refractive index of the waveguide cladding (LC medium around tapered fiber) is obtained [9]. It is possible by changing the external electric field between two electrodes, which is investigated in the next part of the paper.

The TOF-TNLCC consists of a tapered fiber placed between two glass plates covered with ITO and an alignment layer. Biconical optical tapered fiber was manufactured on the dedicated device - fiber optic taper element technology (FOTET). The main advantages of the tapering process are its simplicity and possibility to obtain continuous monitoring of the changes in light beam propagation through the fiber during its tapering. The basic parameters of the process, such as length and velocity of fiber elongation, are controlled by special software. Attenuations of the tapering process are constantly monitored by using a laser $(\lambda=1550 \mathrm{~nm}$, $\mathrm{P}=1.5 \mathrm{~mW}$ ) and a detector connected to the opposite ends of the fiber.

FOTET makes it possible to obtain various types of biconical tapers from different kinds of optical fiber $[10 \div 11]$. In this investigation, the used tapered fiber was made from a telecommunications single mode fiber operating at $\lambda=1550 \mathrm{~nm}$. The elongation obtained by the tapering process was equal to $\mathrm{L}=(20.0 \pm 0.2) \mathrm{mm}$ and the taper waist diameter was $\varphi=(12.0 \pm 0.5) \mu \mathrm{m}$. The applied tapered fiber is characterized by losses lower than $0.5 \mathrm{~dB}$ in the whole investigated spectrum range. Figure 1a presents the scheme of the prepared TOF-TNLCC.

a)

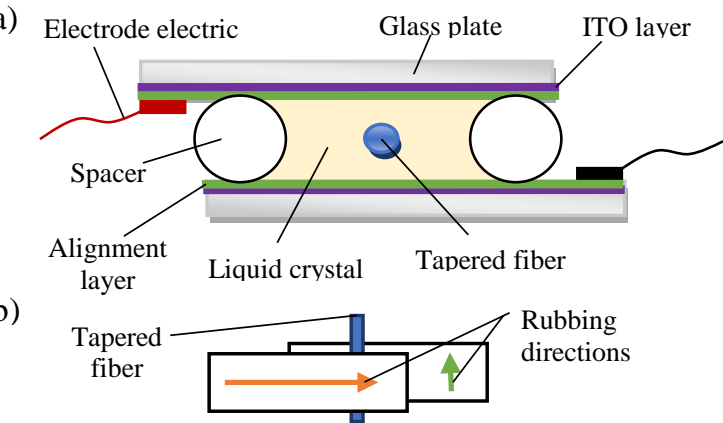

Fig. 1. a) Scheme of the TOF-LCC; b) twisted type of used rubbing. 
In contrast, the cells where the top and bottom electrodes have the same rubbing direction and LC molecules have approximately the same direction in the whole volume. In the twisted cells around the taper waist region, the molecules are changing their orientation in the volume of an LC cell which directly influences propagation.

Measurements were carried out in two measuring systems with different light-sources and detectors. The first one consists of a broad spectrum light source Supercontinuum SuperK EXTREME (SC) and Optical Spectrum Analyzer Yokogawa AQ6373 (OSA) as a detector. The second arrangement was equipped with the laser $\lambda=630 \mathrm{~nm}$ and a photodetector connected to the oscilloscope. The prepared cell was placed into the climatic chamber VLC 7010 and the electric signal was triggered by generator Rigol DG-1032. The sScheme of the measured system is presented in Fig. 2.

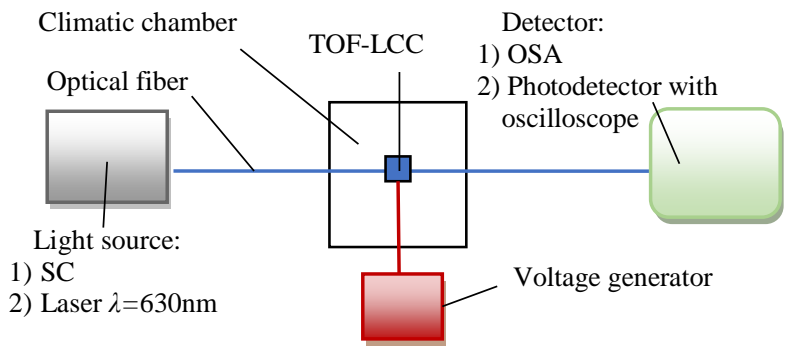

Fig. 2. Scheme of the measured system.

The first measurement was performed for two temperatures $\mathrm{T}=20^{\circ} \mathrm{C}$ and $60^{\circ} \mathrm{C}$ and a different amplitude of applied electric field from 0 to $160 \mathrm{~V}$. The results have been observed in a wide spectral range of used SC $(\lambda=$ $550 \div 1100 \mathrm{~nm}$ ). Comparing the spectra obtained at $\mathrm{T}=20^{\circ} \mathrm{C}$ (Fig. 3) it can be noticed that transmission for a modulated signal (orange line) is placed between transmission without an electrical field (green line) and TOF-LCC transmission for a constant electric field (blue line). Physically, applied modulation causes a decrease in power during n- director reorientation, which corresponds to switching ON and switching OFF. As can be observed, together with an increase in temperature to $\mathrm{T}=60^{\circ} \mathrm{C}$, optical power increases too. Despite the obtained transmission for $\mathrm{T}=60^{\circ} \mathrm{C}$, which is the highest (yellow line), the LC reaches its temperature of isotropic phase transition and further steering of LC molecules by the electric field is impossible. Also, it should be mentioned that due to the increase of steering temperature, the range of transmitted light is broadening over $200 \mathrm{~nm}$.

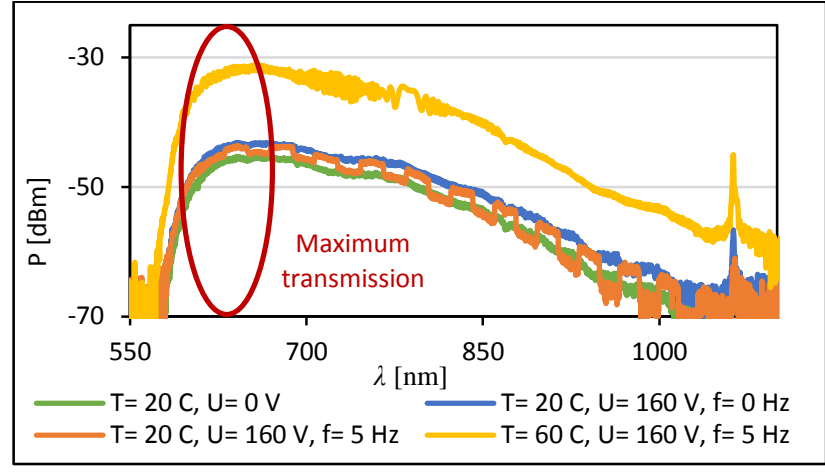

Fig. 3. Transmission in the TOF-LCC at T $=20^{\circ} \mathrm{C}$ and $60^{\circ} \mathrm{C}$ under applied voltage $\mathrm{U}=160 \mathrm{~V}$ with and without modulation $\mathrm{f}=5 \mathrm{~Hz}$.

Maximum transmission for all curves is observed for the wavelength range $\lambda=600 \div 650 \mathrm{~nm}$, hence the second experiment was performed for the wavelength $\lambda=630 \mathrm{~nm}$. The TOF-TNLCC placed in the climatic chamber was heated to $\mathrm{T}=60^{\circ} \mathrm{C}$ and stabilized for one hour. All measurements were performed while the chamber with TOF-TNLCC was slowly cooled down $\left(0.5^{\circ} \mathrm{C} / \mathrm{min}\right)$ from temperature $\mathrm{T}=60^{\circ} \mathrm{C}$ to $\mathrm{T}=20^{\circ} \mathrm{C}$. The photodetector used in the second measurement system converts the optical signal from TOF-TNLCC to a voltage signal, therefore the results are presented as a voltage/time dependence. The obtained results show the same relation between power and temperature as described above - a higher temperature corresponds to a higher signal (transmission) (Fig.4). Also, as it can be observed (Fig. 4a), threshold voltage decreases together with enhanced temperature. The signal at $\mathrm{T}=50^{\circ} \mathrm{C}$ is much higher than for $\mathrm{T}=20^{\circ} \mathrm{C}$.
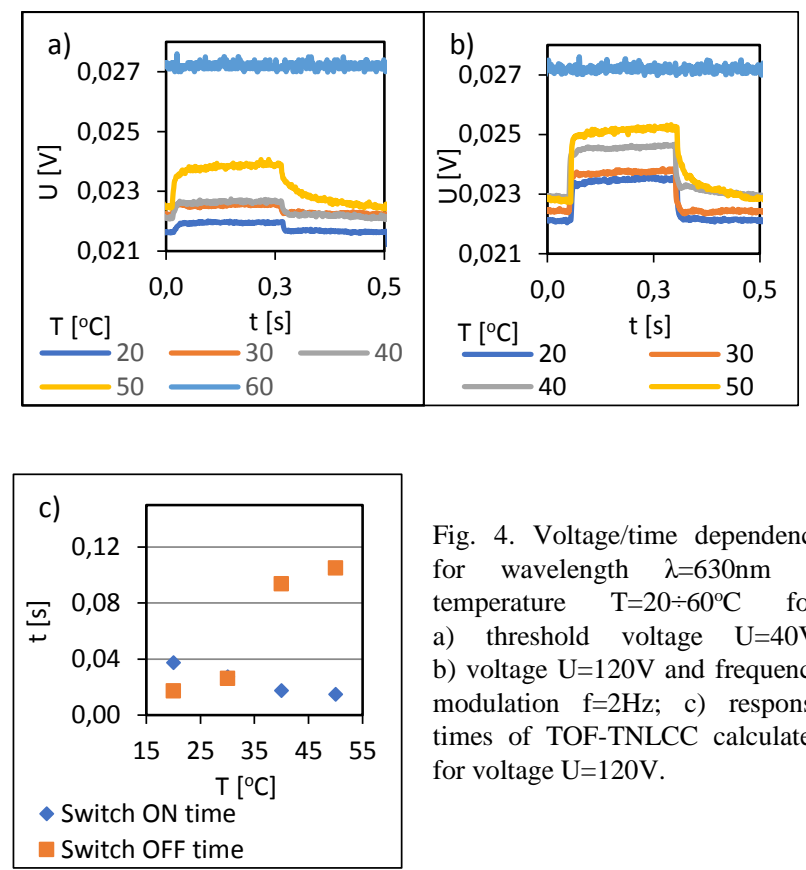

Fig. 4. Voltage/time dependence for wavelength $\lambda=630 \mathrm{~nm}$ at temperature $\mathrm{T}=20 \div 60^{\circ} \mathrm{C}$ for: a) threshold voltage $\mathrm{U}=40 \mathrm{~V}$; b) voltage $U=120 \mathrm{~V}$ and frequency modulation $\mathrm{f}=2 \mathrm{~Hz} ; \mathrm{c}$ ) response times of TOF-TNLCC calculated for voltage $U=120 \mathrm{~V}$. 
This phenomenon is related to the decrease of a liquid crystal refractive index together with increasing temperature. Figure $4 \mathrm{c}$ presents the results regarding the investigated influence of temperature on switch-ON and switch-OFF times. As one can see, the switching ON time decreases, while the switching OFF time increases with temperature. Those relations are connected with the change of liquid crystal physical properties [13]. In the first case, it is related to decreasing liquid crystal viscosity. Mostly, higher viscosity corresponds to higher intermolecular forces, hence the switch $\mathrm{ON}$ time at $\mathrm{T}=20^{\circ} \mathrm{C}$ is longer than at $\mathrm{T}=50^{\circ} \mathrm{C}$. In the second case, the process of relaxation back to the initial orientation is a viscoelastic process and does not depend on voltage, also the order parameter decreases as the temperature increases, thus the switching OFF time is longer.

Additional measurements performed at room temperature show the relations between the shape of the modulated electric signal and TOF-TNLCC answer. Figure 5 presents the results obtained for steering voltage $\mathrm{U}=100 \mathrm{~V}$ and $\mathrm{f}=10 \mathrm{~Hz}$ for differing modulations shapes. As can be observed, the transmission in the optical fiber is changing due to the frequency modulation type: square, sin, and ramp, thus the propagating wave can be modulated in the taper waist region.

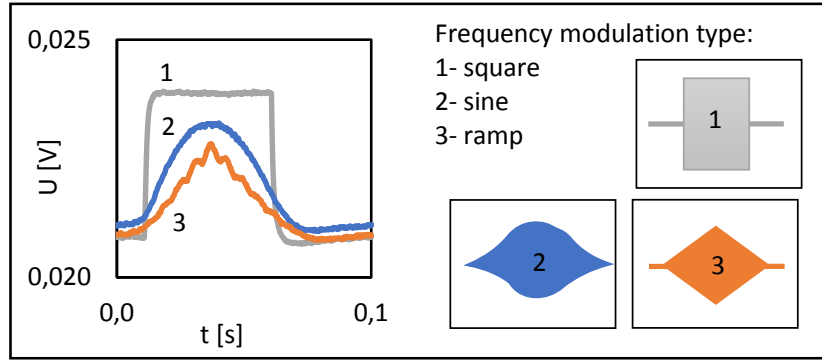

Fig. 5. Voltage/time dependence for different types of frequency modulation for $\mathrm{U}=100 \mathrm{~V}, \mathrm{f}=10 \mathrm{~Hz}$ at $\mathrm{T}=20^{\circ} \mathrm{C}$.

The aim of this paper was to establish the influence of a temperature increase on the spectra of tapered optical fiber with a liquid crystal cladding. It has been stated that together with increasing temperature, optical power passing through the investigated hybrid device increases, which is connected with a decrease in the liquid crystal refractive index and an increase in temperature. In this way, with higher temperature the investigated hybrid structure guides light better. Also, the applied voltage causes an increase in transmission in a wide range of wavelengths. Additional modulation of an applied electric field causes dynamic switching of a liquid crystal.

The obtained results indicate a strong relation between the response time of $\mathrm{LC}$ and temperature of the environment, which is related to changing physical properties of the investigated E7 mixture.
The new hybrid device presented in this paper shows the possibility of applying the sensing techniques (change of temperature, voltage or vibration) in which a propagating wave can be modulated in the taper waist region. Additionally, this device gives the capability for modifying the properties of LC by various types of dopants, e.g. nanoparticles, polymers or other materials which will be more sensitive to magnetic and electric fields or pressure, etc.

This work was supported by the Ministry of National Defense, Republic of Poland Program Research Grant MUT project no. 13-995.

\section{References}

[1] V.J. Tekippe, Proc. SPIE 1085 (1990).

[2] T.A. Birks, Y.W. Li, J. Lightwave Technol. 10, 4 (1992).

[3] J. Korec, K.A. Stasiewicz, O. Strzeżysz, P. Kula, L.R. Jaroszewicz, J. Sensors 2019: 1-11 (2019).

[4] Ch. Veilleux, J. Lapierre, J. Bures, Opt. Lett. 11, 733 (1986).

[5] J.F. Henninot, D. Louvergneaux, N. Tabiryan, M. Warenghem, Mol. Cryst. Liquid Cryst. 282, 297 (1996).

[6] Y. Wang et.al., Opt. Expr. 25, 918 (2017).

[7] J. Korec, K. A. Stasiewicz, O. Strzeżysz, P. Kula, L. R. Jaroszewicz, . E. Moś, Tapered fibre liquid crystal optical device, Proc. SPIE 10681 (2018)

[8] G. Assanto, A. Picardi, R. Barboza, A. Alberucci, Phot. Lett. Poland 4, 1 (2012).

[9] A.Ghanadzadeh Gilani, M.S. Beevers, J. Mol. Liq. 92, 3 (2001).

[10] E.C. Mägi, P. Steinvurzel, B.J. Eggleton, Opt. Expr. 784, 12 (2004).

[11] Y. Li and J. Lit, J. Opt. Soc. Am. A 2, 462 (1985).

[12] J. Korec, K.A. Stasiewicz, L.R. Jaroszewicz, Proc. SPIE 11045, 110450I (2019).

[13] L.M. Blinov, Liquid crystals: physical properties and their possibilities in application in Advances in Liquid Crystal Research and Applications (1981). 